\title{
Changes in the Density of Nitrergic Neurons in the Hippocampus of Rats Following Kainic Acid and Melatonin Administration
}

\author{
A. JAIN ${ }^{1}$, D. SHARMA ${ }^{1}$, P. SUHALKA ${ }^{1}$, P. SUKHWAL ${ }^{1}$, M. BHATNAGAR ${ }^{1}$ \\ ${ }^{1}$ Department of Zoology, University College of Science, Mohan Lal Sukhadia University, Udaipur, \\ Rajasthan, India
}

Received December 5, 2011

Accepted October 26, 2012

On-line December 13, 2012

\begin{abstract}
Summary
Nitric oxide (NO) may play a role in the pathophysiology of excitotoxicity. It is also possible that increase in $\mathrm{Ca}^{2+}$ overload and NO-mediated events are involved in neuronal loss during excitotoxicity. Using nicotinamide adenine dinucleotide phosphate-diaphorase (NADPH-d) histochemistry, we have investigated the effects of melatonin on NADPH-d positive hippocampal neurons after kainic acid (KA) induced excitotoxicity in female rats of Wistar strain. Cytosolic $\mathrm{Ca}^{2+}$ (free calcium) in all the respective experimental groups was also studied. Kainic acid was administered, with a single dose of $10 \mathrm{mg} / \mathrm{kg} / \mathrm{bw}$ (body weight) to the animals. KA treated rats were given melatonin at a dose of $20 \mathrm{mg} / \mathrm{kg} / \mathrm{bw}$ (for 14 day). On the last day of treatment, animals were transcardially perfused with $4 \%$ paraformaldehyde under deep thiopental anaesthesia. Cryostat sections $(20 \mu \mathrm{m})$ were cut and stained for NADPH-d positive neurons. KA exposed animals showed a significantly increased number of NADPH-d positive neurons in the dorsal and ventral blade of the dentate gyrus (DG), hilus, CA1 and CA3 area of hippocampus, with a parallel increase in intracellular free $\mathrm{Ca}^{2+}$ ion concentration, as compared to the control group. KA + melatonin-treated animal groups showed reduced number of NADPH-d positive neurons in DG, hilus, CA1 and CA3 areas and a decline in cytosolic $\mathrm{Ca}^{2+}$ concentration, as compared to $\mathrm{KA}$ treated group. Our study suggests that the enhanced levels of cytosolic $\mathrm{Ca}^{2+}$ and nitric oxide (NO) play an important role in kainate induced excitotoxicity. Inhibition of NO production may be another means whereby melatonin can reduce oxidative damage and seems to play important role in neuroprotection.
\end{abstract}

\section{Key words}

NADPH-diaphorase • Kainic acid • Melatonin • Nitric oxide • Hippocampus

\section{Corresponding author}

M. Bhatnagar, Department of Zoology, University College of Science, M. L. Sukhadia University, Udaipur-313001, India. E-mail: mbhatnagar@yahoo.com or ayushibiotec@yahoo.co.in

\section{Introduction}

During excitotoxicity different pathways are activated, involving elevated free radicals and ROS, rise in intracellular calcium, loss of membrane integrity, progressive proteolysis, and neuronal loss. The alterations due to excitotoxicity lead to nitric oxide synthase (NOS) activation, resulting in enhanced nitric oxide (NO) production (Christopherson and Bredt 1997). NO takes part in the inflammatory and apoptotic pathways (Farooqui et al. 2001). NO has been implicated in the pathophysiology of some neurodegenerative diseases such as Parkinson's (Hantraye et al. 1996), in brain ischemia (Samdani et al. 1997) and epilepsy (Chuang et al. 2009).

NO acts as a neurotransmitter and is also involved in synaptic plasticity, neurite formation and dendritic branching (Bredt 1999). The production of NO in intact neurons occurs in response to excitatory stimuli that requires an intracellular $\mathrm{Ca}^{2+}$ ion influx (Kiss 2000). NO reacts with superoxide radical $\left(\mathrm{O}_{2}^{-}\right)^{-}$, yielding peroxynitrite radical $\left(\mathrm{NOO}^{-}\right)$and eventually hydroxyl ion $\left(\mathrm{HO}^{\circ}\right)$, which are highly reactive free radical species; and the intermediate products which are powerful inducers of lipid, protein, and DNA per oxidation as well (Moncada et al. 1991). An excessive amount of NO, a free radical which is generated by the inducible form of NO synthase, is known to cause cytotoxic changes in cells (Calabrese et 
al. 2007). Hence, NO synthase is considered a prooxidative enzyme, and any factor that reduces its activity would be considered an antioxidant.

Nitric oxide $(\mathrm{NO})$ is produced from L-arginine by NOS. Three different forms of NOS are known, eNOS (endothelial nitric oxide synthase) an endothelial form is responsible for cardiovascular actions, iNOS (inducible nitric oxide synthase) an inducible form found originally in macrophages and involved mainly in immunological processes (Benešová et al. 2005), and nNOS (neuronal nitric oxide synthase) which is a neuronal form. Although all forms can be found in the CNS, the specific actions attributed primarily to $\mathrm{NO}$ production are to nNOS. nNOS (NOS type I) is related with signal transduction in peripheral and central neurons. It is expressed by only a small percentage of neurons, considered to be interneurons. nNOS activity is dependent on intracellular calcium and its production is a calmodulin-dependent process, therefore it must be preceded by elevation of intracellular $\mathrm{Ca}^{2+}$ concentration (Kiss 2000). $\mathrm{Ca}^{2+}$ influx is induced by activation of kainate receptors, in excitotoxicity ( $\mathrm{Lu}$ et al. 1996). Rise in $\mathrm{Ca}^{2+}$ concentration in neurons may eventually lead to the increased excitability, further leading to the activation of nNOS. The enhanced release of nitric oxide due to activation of nNOS ultimately leads to cellular toxicity, which may be an important factor for massive neuronal loss.

Earlier studies have shown that nicotinamide adenine dinucleotide phosphate-diaphorase (NADPH-d) may correspond to nNOS, and it is therefore suggested that neurons containing NADPH-d might be capable of producing NO. The hippocampus, particularly CA3 and CA1 areas are especially sensitive to the excitatory and neurotoxic effect of KA and it is the area of its primary action (Ben-Ari et al. 1981). The neural events in the hippocampus have been studied intensively (ZagulskaSzymczak et al. 2001) due to its involvement in a variety of neurodegenerative conditions as well as its probable role in learning and memory. The marked sensitivity of hippocampus to excitotoxic and neurotoxic effects of KA is due to the great density of binding sites for excitatory amino acids in this region (Benešová et al. 2005, Kubova et al. 2001). It has been studied that KA induces neural cell death due to apoptosis as well as by necrosis (Chuang et al. 2009).

Melatonin, the major hormone produced by the pineal gland, is shown to have antioxidant, anticonvulsant and neuroprotective effects (Jain and Bhatnagar 2007). Recent studies have shown that melatonin inhibits the activity of NO synthase, beside it is NO and peroxynitrite scavenging activity (Tapias et al. 2009). Inhibition of NO production may be another means whereby melatonin can reduce oxidative damage and seems to play important role in neuroprotection.

This study is an attempt to investigate the effects of melatonin on NADPH-d positive hippocampal neurons in kainic acid (KA) induced excitotoxicity in rats. Aim of the study was to assess the cytosolic $\mathrm{Ca}^{2+}$ concentration, NOS activity and to investigate role of melatonin in amelioration of KA induced changes on the nitrergic neurons in hippocampus.

\section{Methods}

Adult female rats, 2 months old of Wistar strain (body weight $120 \pm 5 \mathrm{~g}$ ) were used for the experiments. The animals were housed in polypropylene cages at controlled temperature $\left(25 \pm 2{ }^{\circ} \mathrm{C}\right)$ and relative humidity $(60 \pm 5 \%)$ according to standard laboratory protocol. The animals were given food and water ad libitum and were housed in an animal house with a 12-h light/dark cycle. All experiments were approved by IAEC (Institutional Animal Ethical Committee) and the laboratory was approved by Committee for the Purpose of Control and Supervision of Experiments on Animals (CPCSEA), Government of India (Approval no. \#973/ac/06/CPCSEA).

There were 48 animals in all the experimental groups, with 12 in each group. Animal groups used in the experiment: group I - control/saline solution $(0.2 \mathrm{ml})$, group II - a single dose of kainic acid $(10 \mathrm{mg} / \mathrm{kg})$, group III - a single dose of kainic acid $(10 \mathrm{mg} / \mathrm{kg})$ then melatonin $(20 \mathrm{mg} / \mathrm{kg})$ (melatonin was given immediately after KA treatment on the first day and was given a single dose daily for 14 days), and group IV - a single dose of melatonin $(20 \mathrm{mg} / \mathrm{kg})$ for 14 days. Each injection was intraperitoneal and in the same volume $(0.2 \mathrm{ml})$. Only those rats exhibiting full limbic seizures, forelimb clonus with rearing, were included in the study. Seizures occurred 45-50 min after KA injection. On average, the survival was $66 \%$ in the KA treated group ( 8 rats survived) and in melatonin + KA treated group, it was $83 \%$ (10 rats survived). KA was dissolved in phosphatebuffered saline (PBS), and the $\mathrm{pH}$ adjusted to 7.4 with $\mathrm{NaOH}$. Melatonin was dissolved in absolute ethanol and further diluted in saline to a final ethanol concentration of $2 \%$ (Chung and Han 2003). Melatonin injection was intraperitoneal and in the same volume $(0.2 \mathrm{ml})$. 
All the included animals were decapitated on the 14th day under diethyl ether anesthesia. NADPH-d histochemistry was carried out by the method of SchererSingler et al. (1983). Briefly, brain of control and experimental groups were dissected out, fixed and placed in sucrose solution overnight in each treatment $(10 \%$, $20 \%$ and $30 \%$ respectively). We studied the hippocampus region between the AP plane $2.5 \mathrm{~mm}$ and $4.0 \mathrm{~mm}$ posterior to the bregma. Serial sections were obtained of $20 \mu \mathrm{m}$ thickness on cryostat. Every third section was selected for quantification of NADPH-d positive neurons. 20-25 sections were quantified from each brain. Brain sections were incubated in $0.1 \mathrm{M}$ Phosphate buffer $(\mathrm{pH}=7.4$ ), containing $0.2 \mathrm{mg} / \mathrm{ml}$ nitro blue tetrazolium, $25 \mathrm{mg} / \mathrm{ml}$ NADPH and $0.6 \%$ Triton. Sections were mounted in glycerine jelly. NADPH-d positive neurons were then quantified per section area, under a light microscope (Olympus Provis AX 70) in the CA1, CA3, and DG regions of hippocampal formation. The effect of KA was studied in two distinct hippocampal regions: in the dentate gyrus (DG) and cornu amonis (CA) subfields.

Cytosolic free $\mathrm{Ca}^{2+}$ was measured by using Fluo-3 AM fluorescent dye and by the method of Mattson et al. (1993). Briefly, brain homogenate was centrifuged at $1000 \mathrm{~g}$ and supernatant was then incubated with $5 \mu \mathrm{M}$ Fluo-3 AM for $30 \mathrm{~min}$ in dark. Fluorescence was then measured spectrofluorimetrically at $506 \mathrm{~nm}$ excitation and $526 \mathrm{~nm}$ emission. Free cytosolic $\mathrm{Ca}^{2+}$ was expressed as AFU (arbitrary fluorescence units).

Results were expressed as the mean $\pm \mathrm{SD}$. For the statistical calculation data were analyzed using ANOVA (one way analysis of variance) followed by Bonferroni post test to compare all pairs of groups. Level of significance was set at $\mathrm{p}<0.05$. Prism Software program (Graphpad Software Inc.) was used.

\section{Results}

Systemic KA administration produced the well described sequential behavioral changes (Sperk 1994). Initially the rats exhibited loss of mobility and rigid postures. After 45-50 min, they exhibited changes like staring spells, head nodding, clonic convulsion with rearing and falling. In the present study, co-treatment with melatonin did not significantly alter the clinical course of KA induced limbic seizures.

The results show that kainic acid treatment lowered the number of NADPH-d positive neurons

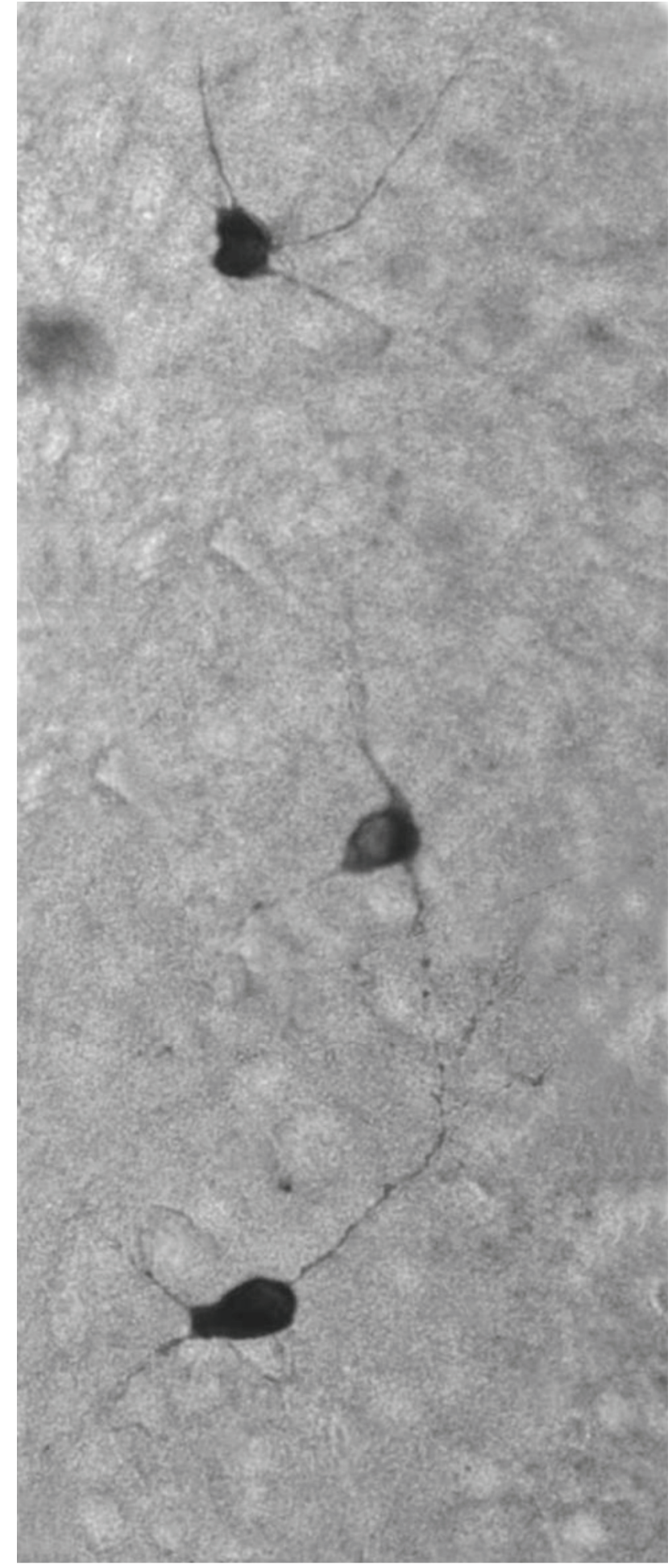

Fig. 1. NADPH-d positive interneuron in the CA1 region of hippocampus. NADPH-d staining. Direct magnification 400x.

(Fig. 1) in all the investigated areas of the hippocampus, compared to the control group which was considered as baseline (Fig. 2A-2E). A significant increase in the intracellular $\mathrm{Ca}^{2+}$ concentration $(\mathrm{p}<0.05)$ in $\mathrm{KA}$ exposed animals, was also observed when compared to the control 
$\mathbf{A}$

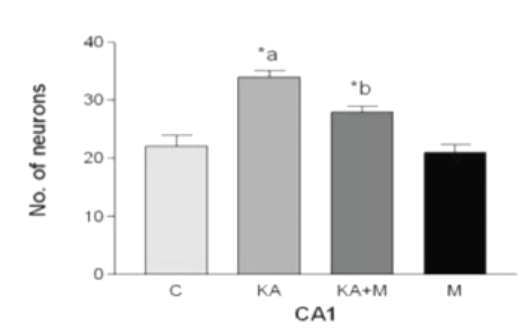

C

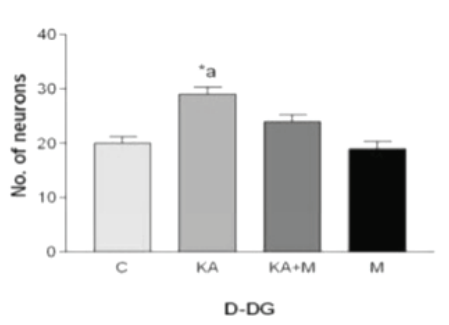

E

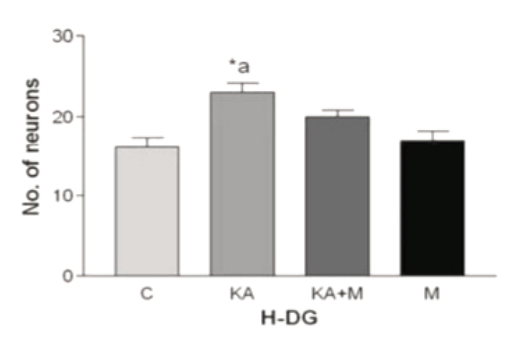

B

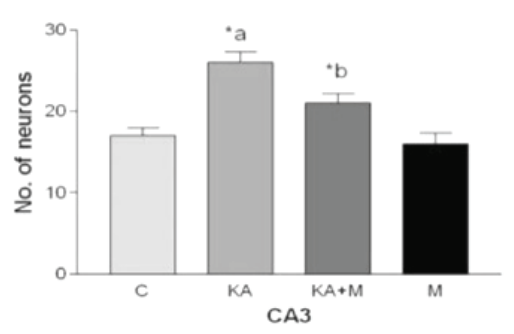

D

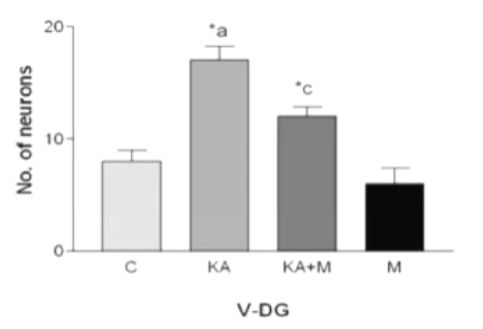

F

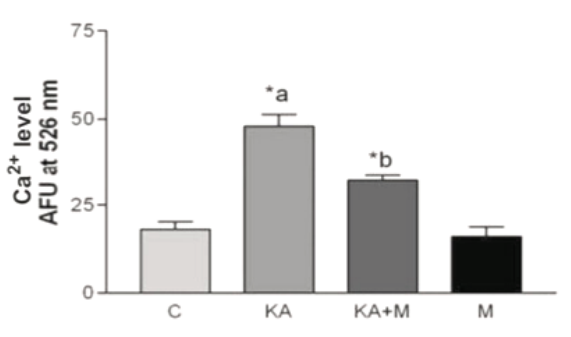

Fig. 2. NADPH-d histochemistry in rat hippocampus (C - Control, KA - kainic acid treated, KA+MEL kainic acid + melatonin-treated and $\mathrm{M}$ - melatonin-treated). A: Number of NADPH-d positive neurons in the CA1 region of hippocampus per section area. B: Number of NADPH-d positive neurons in the CA3 region of hippocampus per section area. C: Number of NADPH-d positive neurons in the dorsal blade of dentate gyrus per section area. D: Number of NADPH-d positive neurons in the ventral blade of dentate gyrus per section area. E: Number of NADPH-d positive neurons in the hilus of dentate gyrus per section area. F: Free cytosolic $\mathrm{Ca}^{2+}$ level measured by using Fluo-3 AM, $\mathrm{Ca}^{2+}$ concentration is measured arbitrarily by determining fluorescence units. All values are expressed as mean $\pm \mathrm{SD}$, data comparisons were carried out using one way analysis of variance followed by Bonferroni post test to compare all pairs of groups, $(p<0.05) . *-$ difference is significant at $5 \%$ level of probability, *a - when compared with control group, *b - when compared with KA-treated group, ${ }^{*} \mathrm{C}$ - when compared with $\mathrm{KA}+\mathrm{M}$ treated group. group (Fig. 2F). Melatonin treatment successfully prevented the rise in number of NADPH-d positive neurons in hippocampus. Melatonin treatment in group III, significantly $(p<0.05)$ decreased the number of NADPH-d positive neurons in CA1 (Fig. 2A), CA3 (Fig. 2B), and DG (Fig. 2C, 2D) regions of the hippocampus, when compared to the KA treated group II. Melatonin treatment after KA exposure in group III, showed a significant $(\mathrm{p}<0.05)$ decline in the elevated cytosolic $\mathrm{Ca}^{2+}$ concentration, when compared to KA treated animals indicating the possible role of melatonin in calcium homeostasis (Fig. 2F).

In the present study, treatment with melatonin did not significantly alter the number of NADPH-d positive neurons in group IV. Results show that this group treated with melatonin alone did not show any significant difference in the number of NADPH-d positive neurons in all investigated regions of the hippocampus, when compared to the control group. No significant change in free cytosolic calcium ion concentration was recorded when compared to the control group (Fig. 2F).

\section{Discussion}

In this investigation, we demonstrated that treatment with kainic acid increased the number of NADPH-d positive neurons, in all examined areas of the hippocampus. It is evident that nNOS activity in kainate treated group is not regionally homogenous, hippocampal CA1 and CA3; regions show enhanced production of NO when compared to the control group. Our data were consistent with previous reports showing that the CA1 and CA3 zones, which are most susceptible to neuronal injury following KA treatment, never recovered protein synthesis, indicating that a prolonged deficit in protein synthesis correlates with selective vulnerability of different nuclei (White et al. 2000, Farooqui et al. 2001, Benešová et al. 2005). These finding are consistent with our report that show hippocampal neurons to be the most vulnerable to KA toxicity. This also support the view that KA causes a rise in the level of ROS, a rapid increase in $\mathrm{Ca}^{2+}$ overload, enhanced NO production, generalized depolarization, progressive proteolysis, and loss of membrane integrity, which ultimately result into neurodegenration (Montecot 
et al. 1997, Benešová et al. 2004).

While KA has been widely used as a model of temporal lobe epilepsy and selective hippocampal neurodegenration, few attempts have been made to characterize the role of calcium imbalance and nNOS activity associated with this limbic seizure model. Although the mechanism of neurotoxic effect of $\mathrm{NO}$ in KA-induced injury is not clearly known, it is attributed to the oxidative effects and calcium imbalance.

Our results show that melatonin co-treatment against KA toxicity, decreased the density of nitrergic elements in all the examined hippocampal sub regions. Melatonin administration decreased nNOS activity, calcium overload and kainate induced neurodegenration. The underlying mechanism of neuroprotection by melatonin is attributed to its potent free radical scavenging ability and maintenance of calcium homeostasis (Jain and Bhatnagar 2007, Tapias et al. 2009). Furthermore melatonin inhibits the production of $\mathrm{NO}$ and may contribute to its neuroprotective properties in various pathophysiological conditions (Chung and Han 2003). Many studies have shown that nicotinamide adenine dinucleotide phosphate-diaphorase (NADPH-d) may correspond to the neuronal NOS, and it is therefore suggested that neurons containing NADPH-d are likely to be capable of producing NO (Montecot et al. 1997). The method used to demonstrate nitrergic elements in the brain is based on histochemical reaction for NADPH-d. The relatively simple NADPH-d histochemical technique was widely used to identify NO producing elements in the brain of representatives of all vertebrate classes (Benešová et al. 2005).

It has been repeatedly proved that NADPH-d activity and NOS immunoreactivity in the nervous system is widely co-localized in the same sets of neurons (Moreno et al. 2002). KA administration brought about enhanced release of $\mathrm{NO}$ in all examined regions of the hippocampus which was significantly observed in CA1, CA3 areas of the hippocampus causing maximum injury to the neurons. The principal cell type of CA1 and CA3 regions is the pyramidal cell (Lorente de Nó 1934). CA3 pyramidal neurons are among the most responsive neurons to kainate in the brain (Ben-Ari and Cossart 2000). It is well established that prolonged seizure activity can lead to irreversible brain damage, by both necrotic and apoptotic types of cell death, which has been recently reported as a consequence of seizures (Langmeier et al. 2003). Intraperitoneal administration of
KA results in the death of neurons in the CA1 and CA3 areas due to excitotoxicity as evident from our results. The altered cellular processes caused by KA administration include: enhanced ROS production, excessive $\mathrm{Ca}^{2+}$ overload, NOS activation, neuronal cell loss, and glial reactivation (Wojtal et al. 2003).

The number of NADPH-d positive neurons in dorsal and ventral blades of the dentate gyrus increased after KA administration but these changes were not as evident as in CA1 and CA3 regions of the hippocampus. Our results, showing a regulatory effect of melatonin on NOS activity and intracellular $\mathrm{Ca}^{2+}$ could be explained by melatonin's neuroprotective role. It is a potent free radical scavenger, an antioxidant that protects cells against the damage induced in different pathologic conditions (Chung and Han 2003). NADPH-d positive neurons are probably interneurons. The reason of the alteration in number of NADPH-d neurons can also be a result of changes in gene expression by melatonin (Kotler et al. 1998). Our findings also suggest that $\mathrm{Ca}^{2+}$ overload induced higher expression of nNOS, which could contributes to the sustained neuronal excitation and ultimately in enhanced activity of nNOS. In fact, NOS gene expression activated by hypoxia in central and peripheral neurons had recently been observed (Chang et al. 2003).

In conclusion, this study provides evidence that melatonin posses neuroprotective properties against KAinduced toxicity, and indicates its efficiency to regulate $\mathrm{Ca}^{2+}$ and NO levels in different regions of hippocampus. The changes in the density of nitrergic neurons in KA and melatonin exposed animals are region specific in rat brain. Based on our findings, it seems that melatonin has an important role to play and regulate neuronal loss in excitotoxicity.

\section{Conflict of Interest}

There is no conflict of interest.

\section{Abbreviations}

NADPH-d - nicotinamide adenine dinucleotide phosphate-diaphorase; $\quad \mathrm{NO}-$ nitric oxide; $\mathrm{Ca}^{2+}-$ free calcium ion; KA - kainic acid; ROS - reactive oxygen species, mg - milligram; kg - kilogram; bw - body weight; $\mu \mathrm{m}$ - micrometer; DG - Dentate Gyrus; CA1 cornu ammonis 1; CA3 - cornu ammonis; CNS - central nervous system; NOS - nitric oxide synthase; eNOS endothelial nitric oxide synthase; iNOS - inducible nitric oxide synthase; nNOS - neuronal nitric oxide synthase. 


\section{References}

BEN-ARI Y, COSSART R: Kainate, a double agent that generates seizures: two decades of progress. Trends Neurosci 23: $580-587,2000$.

BEN-ARI Y, TREMBLAY E, RICHE D, GHILINI G, NAQUET R: Electrographic, clinical and pathological alterations following systemic administration of kainic acid, bicuculline or pentetrazole: metabolic mapping using the deoxyglucose method with special reference to the pathology of epilepsy. Neuroscience 6: 13611391, 1981.

BENEŠOVÁ P, LANGMEIER M, BETKA J, TROJAN S: Changes in the number of nitrergic neurons following kainic acid administration and repeated long-term hypoxia. Physiol Res 53: 343-349, 2004.

BENEŠOVÁ P, LANGMEIER M, BETKA J, TROJAN S: Long-lasting changes in the density of nitrergic neurons following kainic acid administration and chronic hypoxia. Physiol Res 54: 565-571, 2005.

BREDT DS: Endogenous nitric oxide synthesis: biological functions and pathophysiology. Free Radic Res 31: 577596, 1999.

CALABRESE V, MANCUSO C, CALVANI M, RIZZARELLI E, BUTTERFIELD DA, STELLA AM: Nitric oxide in the central nervous system: neuroprotection versus neurotoxicity. Nat Rev Neurosci 8: 766-775, 2007.

CHANG HM, LIAO WC, LUE JH, WEN CY, SHIEH JY: Upregulation of NMDA receptor and neuronal NADPHd/NOS expression in the nodose ganglion of acute hypoxic rats. J Chem Neuroanat 25: 137-147, 2003.

CHRISTOPHERSON KS, BREDT DS: Nitric oxide in excitable tissue: physiological roles and disease. $J$ Clin Invest 100: 2424-2429, 1997.

CHUANG YC, CHEN SD, LIOU CW, LIN TK, CHANG WN, CHAN SH, CHANG AY: Contribution of nitric oxide, superoxide anion, and peroxynitrite to activation of mitochondrial apoptotic signaling in hippocampal CA3 subfield following experimental temporal lobe status epilepticus. Epilepsia 50: 731-746, 2009.

CHUNG SY, HAN SH: Melatonin attenuates kainic acid-induced hippocampal neurodegenration and oxidative stress through microglial inhibition. J Pineal Res 34: 95-102, 2003.

FAROOQUI AA, YI ONG W, LU XR, HALLIWELL B, HORROCKS LA: Neurochemical consequences of kainateinduced toxicity in brain: involvement of arachidonic acid release and prevention of toxicity by phospholipase $\mathrm{A}_{2}$ inhibitors. Brain Res Rev 38: 61-78, 2001.

HANTRAYE P, BROUILLET E, FERRANTE R, PALFI S, DOLAN R, MATTHEWS RT, BEAL MF: Inhibition of neuronal nitric oxide synthase prevents MPTP-induced parkinsonism in baboons. Nat Med 2: 1017-1021, 1996.

JAIN A, BHATNAGAR M: Melatonin - a "Magic Biomolecule". Ann Neurosci 14: 108-114, 2007.

KISS JP: Role of nitric oxide in the regulation of monoaminergic neurotransmission. Brain Res Bull 52: 459-466, 2000.

KOTLER M, RODRIGUEZ C, SAINZ RM, ANTOLIN I, MENÉNDEZ-PELÁEZ A: Melatonin increases gene expression for antioxidant enzymes in rat brain cortex. J Pineal Res 24: 83-89, 1998.

KUBOVÁ H, MIKULECKÁ A, HAUGVICOVÁ R, LANGMEIER M, MAREŠ P: Nonconvulsive seizures result in behavioral but not electrophysiological changes in developing rats. Epilepsy Behav 2: 473-480, 2001.

LANGMEIER M, FOLBERGROVÁ J, HAUGVICOVÁ R, POKORNÝ J, MAREŠ P: Neuronal cell death in hippocampus induced by homocysteic acid in immature rats. Epilepsia 44: 299-304, 2003.

LORENTE DE NÓ R: Studies on the structure of the cerebral cortex. II. Continuation of the study of the ammonic system. J Psychol Neurol 46: 113-177, 1934.

LU YM, YIN HZ, CHIANG J, WEISS JH: $\mathrm{Ca}^{2+}$-permeable AMPA/kainate and NMDA channels: high rate of $\mathrm{Ca}^{2+}$ influx underlies potent induction of injury. J Neurosci 16: 5457-5465, 1996.

MATTSON MP, TOMASELLI KJ, RYDEL RE: Calcium destabilizing and neurodegenerative effects of aggregated beta-amyloid peptide are attenuated by basic FGF. Brain Res 621: 35-49, 1993.

MONCADA S, PALMER RM, HIGGS EA: Nitric oxide: physiology, pathophysiology and pharmacology. Pharmacol Rev 43: 109-142, 1991.

MONTECOT C, BORREDON J, SEYLAZ J, PINARD E: Nitric oxide of neuronal origin is involved in cerebral blood flow increase during seizures induced by kainate. J Cereb Blood Flow Metab 17: 94-99, 1997. 
MORENO N, LÓPEZ JM, SÁNCHEZ-CAMACHO C, GONZÁLEZ A: Development of NADPH-diaphorase/nitric oxide synthase in the brain of the urodele amphibian Pleurodeles waltl. J Chem Neuroanat 23: 105-121, 2002.

SAMDANI AF, DAWSON TM, DAWSON VL: Nitric oxide synthase in models of focal ischemia. Stroke 28: 12831288, 1997.

SCHERER-SINGLER U, VINCET SR, KIMURA H, MCGEER EG: Demonstration of a unique population of neurons with NADH diaphorase histochemistry. J Neurosci Methods 9: 229-234, 1983.

SPERK G: Kainic acid seizures in the rat. Prog Neurobiol 42: 1-32, 1994.

TAPIAS V, ESCAMES G, LÓPEZ LC, LÓPEZ A, CAMACHO E, CARRIÓN MD, ENTRENA A, GALLO MA, ESPINOSA A, ACUNA-CASTROVIEJO D: Melatonin and its brain metabolite N(1)-acetyl-5methoxykynuramine prevent mitochondrial nitric oxide synthase induction in parkinsonian mice. $J$ Neurosci Res 13: 3002-3010, 2009.

WHITE BC, SULLIVAN JM, DEGRACIA DJ, O’NEIL BJ, NEUMAR RW, GROSSMAN LI, RAFOLS JA, KRAUSE GS: Brain ischemia and reperfusion: molecular mechanisms of neuronal injury. $J$ Neurol Sci 179: 1-33, 2000.

WOJTAL K, GNIATKOWSKA-NOWAKOWSKA A, CZUCZWAR SJ: Is nitric oxide involved in the anticonvulsant action of antiepileptic drugs? Pol J Pharmacol 55: 535-542, 2003.

ZAGULSKA-SZYMCZAK S, FILIPKOWSKI RK, KACZMAREK L: Kainate induced genes in the hippocampus: lessons from expression patterns. Neurochem Int 38: 485-501, 2001. 